\title{
Stability of Gain Scheduling Control for Aircraft with Highly Nonlinear Behavior
}

\author{
Fany Mendez-Vergara, Ilse Cervantes, and Angelica Mendoza-Torres \\ Hybrid Systems Laboratory, Institute for Scientific and Technological Research of San Luis Potosi (IPICyT), \\ Camino a la Presa San Jose 2055, Colonia Lomas 4ta, 78216 San Luis Potosi, SLP, Mexico \\ Correspondence should be addressed to Ilse Cervantes; ilse@ipicyt.edu.mx
}

Received 30 November 2013; Accepted 21 April 2014; Published 21 May 2014

Academic Editor: Sheldon S. Williamson

Copyright (C) 2014 Fany Mendez-Vergara et al. This is an open access article distributed under the Creative Commons Attribution License, which permits unrestricted use, distribution, and reproduction in any medium, provided the original work is properly cited.

\begin{abstract}
The main goal of this work is to study the stability properties of an aircraft with nonlinear behavior, controlled using a gain scheduled approach. An output feedback is proposed which is able to guarantee asymptotical stability of the task-coordinates origin and safety of the operation in the entire flight envelope. The results are derived using theory of hybrid and singular perturbed systems. It is demonstrated that both body velocity and orientation asymptotic tracking can be obtained in spite of nonlinearities and uncertainty. The results are illustrated using numerical simulations in F16 jet.
\end{abstract}

\section{Introduction}

Gain scheduled control (GSC) is widely employed in flight control applications. The principle of operation of such a controller relies on the use of a piece-wise linear approximation of aircraft dynamics to conclude stability via direct or indirect arguments relating the linear approximation and the original nonlinear dynamics [1]. Usually, stability of every linear system is analyzed, and the design of an interpolation controller to guarantee stable switching is performed as a separated control design task [2-4].

Since the flight dynamics have nonlinear nature, the linear approximation of the system leads to controllers with stability and performance limitations. To overcome such limitations there exist in the literature a variety of control methodologies which provide important advances in GSC and hybrid and nonlinear approaches [5-8]; see also GSC surveys [1,9-19].

Robustness of controllers is crucial not only due to the high nonlinear behavior of the system, but also because aeronautical systems are subjected to external perturbations and they must have some fault tolerant capabilities. There exist in the literature a variety of works leading the control of these systems from a robust or adaptive point of view. In
[14] a multi-input, multioutput nonlinear model predictive controller is proposed that makes use of a neurofuzzy predictor model. The authors analyze the robustness of the proposed control in presence of uncertainties in the physical parameters. In [15] attitude stabilization with external disturbance, unknown inertia parameters, and actuator uncertainties is analyzed. An adaptive control is proposed and input-tostate stability is derived. Reference [16] studies the spacecraft attitude tracking problem; the conditions that guarantee that the attitude and body-rate error are ultimately bounded are derived and bounds for the steady-state tracking errors are also provided. Reference [17] proposes an observer-controller control scheme to solve the output feedback attitude control of a rigid body with bounded input.

In [10] a robust sum-of-squares (SOS) LMI (linear matrix inequality) method to design a nonlinear controller for longitudinal dynamics with parametric uncertainties is proposed. The control design problem is formulated as a robust SOS problem and then an LMI representation is derived using an algorithm that is solved via a stochastic ellipsoid method. In [11] an output feedback to stabilize a planar nonlinear model of a vertical take-off and landing (PVTOL) aircraft is proposed. To address the lack of full state measurement, a finite time convergent observer is 
designed. Reference [12] addresses the nonlinear tracking problem of a roll-rate-sensorless vertical take-off and landing aircraft in the presence of roll-angle measurement delay. The authors develop an extended attitude observer and a tracking controller based on the observer by using the first-order Pade approximation signal of the delayed roll angle, two global coordinate transformations, and an extension of the backstepping technique.

In many of the works above, it is not clear if the controller can deal with structural uncertainty that may arise from a failure, or from the nonlinearities of the system. Moreover, some of them make use of observers to estimate the entire state, which usually leads to high-order controllers. Some of these drawbacks have made researches investigate improved GSC strategies that can ensure the stability of the system in spite of nonlinearities but still with simple controllers. Many of these works make use of recent advances in switching and hybrid systems.

Recently, switched LPV (linear parameter varying) systems have gained the attention of researchers due to the fact that stabilization can also be derived using a multiple Lyapunov function approach. In [20] it is studied the GSC using a switched polytopic approach. In such work, it was proved that the switched polytopic system is input-to-state bounded if the subsystems share a common Lyapunov function, and average dwell time is no smaller than a given constant. On the other hand, [21] proposed a hybrid tracking control for aircraft described by discrete-time stochastic linear hybrid systems; the flight logic is described by discrete dynamics governed by Markov chains. Conditions for convergence of a model adaptive estimation algorithm are proposed, and a new residual-mean interacting multiple model algorithm is proposed.

Reference [18] uses a piecewise linear description of the system and variations in operation points modeled as switches between constituent linearized system dynamics to analyze the control tracking problem. An adaptive control scheme is proposed that can achieve asymptotic tracking performance if the reference input is sufficiently rich and the switches are sufficiently slow. Reference [19] develops a hybrid control strategy that provides autonomous transition between hovered and leveled flights to a model-scale fixedwing aircraft. The controller, together with an appropriate choice of reference maneuvers, ensures that a transition from hovered flight to level flight, or vice versa, is achieved. In [22], a sensor-based backstepping method is implemented on a fixed-wing aircraft with its focus on handling structural changes caused by damages. The controller combines nonlinear dynamic inversion, which is highly dependent on the knowledge of the system structure, with a sensor-based backstepping control approach.

In this work, we are focused on the velocity regulation and orientation of nonlinear aircraft. It is known (see [23]) that the more general description of the flight dynamics is piecewise nonlinear since forces and torques involved may vary dramatically with altitude, angle of attack, side-slip angle, and mach number, among others. That is, in the best situation, multiple nonlinear description of the aircraft constitutes the more reliable description that can be obtained. Under this premise, we depart from a six degree of freedom, nonlinear piece-wise description of the aircraft to study the stability of the system under a simple linear dynamic output feedback (as in GSC). Using theory of switched hybrid systems and singular perturbation systems, we demonstrate that the origin of the closed-loop piecewise nonlinear description of the aircraft is asymptotically stable, even in presence of uncertainties that can be either structural or parametric. This result constitutes the main contribution of this paper.

The tracking control strategy proposed here achieves a desired linear velocity and attitude, while ensuring body rate stabilization in spite of nonlinearities and model uncertainty. As in GSC, the proposed controller is able to adapt its operation based on the suitable definition of the switching criterion. Stability conditions for every operation mode and the overall switching system are derived; such piece-wise nonlinear analysis has not been performed before, to the authors' knowledge.

Based on the aircraft movement restrictions, a cascade control structure is proposed to solve the underactuated task of solving simultaneously body rate stabilization and attitude regulation. The attitude problem is formulated using the kinematic restrictions relating angular body velocity and quaternions, avoiding in this way the drawback of multiple singularities in a given operation condition. It is shown that the proposed controller is able to robustly stabilize the origin of the controlled nonlinear dynamics of the aircraft in spite of parametric or structural uncertainties, as long as they satisfy being bounded and continuous Lipschitz functions of their arguments in the operation domain. Numerical simulations of an F16 aircraft are used to illustrate advantages of the proposed control.

The paper begins with the nonlinear piecewise description of the aircraft and the control problem formulation. Section 3 introduces some preliminary results to establish the stability and the equilibrium uniqueness of some auxiliary systems, while Section 4 is devoted to deriving the main result of the paper. Illustrations of the results are given in Section 5 using a fixed-wing aircraft. Finally, Section 6 summarizes the main contribution of the proposed structure and presents some conclusions.

\section{Problem Statement and Dynamic Description of the Aircraft}

In this work, we depart from a nonlinear description of the aircraft using wind axis. As it is known, the nonlinear behavior of the aircraft depends crucially on the movement task performed. Typically the model parameters are obtained about certain operation conditions. Due to this fact, the nonlinear behavior of the aircraft is better described using piecewise continuous models. In [23], it is stated that the nonlinear description of the aircraft depends on variables such as dynamic pressure, altitude, angle of attack, and sideslip angle among the most important. Let the nonlinear 
model $j$ be valid in the domain $\Omega_{j}$. Departing form this premise, the piecewise nonlinear description of the system can be written without loss of generality as constituted by a linear function and a nonlinear function $\left({ }^{j} \bar{\Phi}(x)\right)$ clustering high order terms and uncertainty, depending exclusively on system's states. In this work, we assume that such description is valid on a domain $\Omega_{j}$. In this way, the airplane movement description and orientation is given by

$$
\begin{gathered}
\dot{x}={ }^{j} A x(t)+{ }^{j} B u(t)+{ }^{j} \bar{\Phi}(x), \\
\dot{\vartheta}=T(\vartheta) \omega_{w},
\end{gathered}
$$

$$
\begin{aligned}
& { }^{j} A=m\left[\begin{array}{cccccc}
X_{V}+X_{T_{V}} \cos \left(\alpha_{e}+\alpha_{T}\right) & 0 & X_{\alpha} & 0 & 0 & 0 \\
0 & Y_{\beta} & 0 & Y_{p} & 0 & Y_{r}-V_{T_{e}} \\
Z_{V}-X_{T_{V}}\left(\alpha_{e}+\alpha_{T}\right) & 0 & Z_{\alpha} & 0 & V_{T_{e}}+Z_{q} & 0 \\
0 & \mu L_{\beta}+\sigma_{1} N_{\beta} & 0 & \mu L_{p}+\sigma_{1} N_{p} & 0 & \mu L_{r}+\sigma_{1} N_{r} \\
M_{V}+M_{T_{V}} & 0 & M_{\alpha}+M_{T \alpha} & 0 & M_{q} & 0 \\
0 & \mu N_{\beta}+\sigma_{2} L_{\beta} & 0 & \mu N_{p}+\sigma_{2} L_{p} & 0 & \mu N_{r}+\sigma_{2} L_{r}
\end{array}\right], \\
& { }_{B} B=\left[\begin{array}{cccc}
\frac{1}{\mathrm{~m}} \frac{\partial D_{a}}{\partial \delta_{T}} & \frac{1}{\mathrm{~m}} \frac{\partial D_{a}}{\partial \delta_{e}} & \frac{1}{\mathrm{~m}} \frac{\partial D_{a}}{\partial \delta_{a}} & \frac{1}{\mathrm{~m}} \frac{\partial D_{a}}{\partial \delta_{r}} \\
\frac{1}{\mathrm{~m} V_{T}} \frac{\partial C_{a}}{\partial \delta_{T}} & \frac{1}{\mathrm{~m} V_{T}} \frac{\partial C_{a}}{\partial \delta_{e}} & \frac{1}{\mathrm{~m} V_{T}} \frac{\partial C_{a}}{\partial \delta_{a}} & \frac{1}{\mathrm{~m} V_{T}} \frac{\partial C_{a}}{\partial \delta_{r}} \\
\frac{1}{\mathrm{~m} V_{T} \cos \beta} \frac{\partial L}{\partial \delta_{T}} & \frac{1}{\mathrm{~m} V_{T} \cos \beta} \frac{\partial L}{\partial \delta_{e}} & \frac{1}{\mathrm{~m} V_{T} \cos \beta} \frac{\partial L}{\partial \delta_{a}} & \frac{1}{\mathrm{~m} V_{T} \cos \beta} \frac{\partial L}{\partial \delta_{r}} \\
J_{w}^{-1} \frac{\partial l_{w}}{\partial \delta_{T}} & J_{w}^{-1} \frac{1}{I_{x}^{\prime}} \frac{\partial l_{w}}{\partial \delta_{e}} & J_{w}^{-1} \frac{1}{I_{x}^{\prime}} \frac{\partial l_{w}}{\partial \delta_{a}} & J_{w}^{-1} \frac{1}{I_{x}^{\prime}} \frac{\partial l_{w}}{\partial \delta_{r}} \\
J_{w}^{-1} \frac{\partial m_{w}}{\partial \delta_{T}} & J_{w}^{-1} \frac{1}{I_{x}^{\prime} \frac{\partial m_{w}}{\partial \delta_{e}}} & J_{w}^{-1} \frac{1}{I_{x}^{\prime}} \frac{\partial m_{w}}{\partial \delta_{a}} & J_{w}^{-1} \frac{1}{I_{x}^{\prime}} \frac{\partial m_{w}}{\partial \delta_{r}} \\
J_{w}^{-1} \frac{\partial n_{w}}{\partial \delta_{T}} & J_{w}^{-1} \frac{1}{I_{x}^{\prime} \frac{\partial n_{w}}{\partial \delta_{e}}} & J_{w}^{-1} \frac{1}{I_{x}^{\prime}} \frac{\partial n_{w}}{\partial \delta_{a}} & J_{w}^{-1} \frac{1}{I_{x}^{\prime}} \frac{\partial n_{w}}{\partial \delta_{r}}
\end{array}\right], \\
& j \bar{\Phi}(\mathbf{x})=\left[\begin{array}{c}
\frac{1}{\mathrm{~m}}\left\{D_{a_{e}}+\sum D_{a}\left(V_{T}, \beta, \alpha, P, Q, R\right)-g \cos \alpha \cos \beta \sin \theta+g \sin \beta \sin \phi \cos \theta+g \sin \alpha \cos \phi \cos \theta\right\}+\overline{\varphi_{1}} \\
\left\{\frac{1}{\mathrm{~m} V_{T}}\right\}\left\{\mathrm{m} V_{T} R+C_{a_{e}}+\sum C_{a}\left(V_{T}, \beta, \alpha, P, Q, R\right)+g \cos \alpha \sin \beta \sin \theta\right. \\
+g \cos \beta \sin \phi \cos \theta-g \sin \alpha \sin \beta \cos \phi \cos \theta\}+\overline{\varphi_{2}} \\
\left\{\frac{1}{\mathrm{~m} V_{T} \cos \beta}\right\}\left\{\mathrm{m} V_{T}\{Q \cos \beta-P \sin \beta\}+L_{e}+\sum L\left(V_{T}, \beta, \alpha, P, Q, R\right)+g \sin \alpha \sin \theta\right. \\
\left.+g \cos \alpha \cos \phi \cos \theta+\frac{\partial L}{\partial \delta_{e}} \delta_{e}+\frac{\partial L}{\partial \delta_{r}} \delta_{r}\right\}+\overline{\varphi_{3}} \\
J_{w}^{-1}\left\{P Q I_{x z}^{\prime}+Q R I_{y}^{\prime}+R Q I_{z}^{\prime}+l_{w_{e}}+\sum l_{w}\left(V_{T}, \beta, \alpha, P, Q, R\right)\right\}+\overline{\varphi_{4}} \\
J_{w}^{-1}\left\{-P\left\{R I_{z}^{\prime}+P I_{x z}^{\prime}\right\}-R\left\{P I_{x z}^{\prime}+P I_{z}^{\prime}\right\}+m_{w_{e}}+\sum m_{w}\left(V_{T}, \beta, \alpha, P, Q, R\right)\right\}+\overline{\varphi_{5}} \\
J_{w}^{-1}\left\{P Q I_{z}^{\prime}-Q P I_{y}^{\prime}+R Q I_{x z}^{\prime}+n_{w_{e}}+\sum n_{w}\left(V_{T}, \beta, \alpha, P, Q, R\right)\right\}+\overline{\varphi_{6}}
\end{array}\right],
\end{aligned}
$$

with ${ }^{j} A,{ }^{j} B$, and ${ }^{j} \bar{\Phi}(x)$ given by

where $x=\left[V_{T}, \beta, \alpha, P, Q, R\right]^{T}$ are deviation variables with respect to an operation point, $\vartheta=[\eta, \epsilon]^{T}, \epsilon=\left[\epsilon_{1}, \epsilon_{2}, \epsilon_{3}\right]^{T}$, and $\omega_{w}=[P, Q, R]^{T}$. Moreover,

$$
T(\vartheta) \triangleq\left[\begin{array}{c}
-\frac{1}{2} \epsilon^{T} \\
\frac{1}{2}[\eta I-S(\epsilon)]
\end{array}\right],
$$

where

$$
S(\epsilon)=\left[\begin{array}{ccc}
0 & -\epsilon_{3} & \epsilon_{2} \\
\epsilon_{3} & 0 & -\epsilon_{1} \\
-\epsilon_{2} & \epsilon_{1} & 0
\end{array}\right],
$$


where $\overline{\varphi_{i}} i=1, \ldots, 6$ account for state-dependent uncertainties, and $\sum D_{a}\left(V_{T}, \beta, \alpha, P, Q, R\right), \sum C_{a}\left(V_{T}, \beta, \alpha, P, Q, R\right)$, and so forth, are terms accounting for higher order nonlinear terms as shown in the following example:

$$
\begin{aligned}
\sum D_{a}\left(V_{T}, \beta, \alpha, P, Q, R\right) & \left\{\frac{\partial^{2} D_{a}}{\partial V_{T}^{2}} \frac{V_{T}^{2}}{2 !}+\frac{\partial^{3} V_{T}}{\partial V_{T}^{3}} \frac{V_{T}^{3}}{3 !}+\frac{\partial^{4} V_{T}}{\partial V_{T}^{4}} \frac{V_{T}^{4}}{4 !}+\cdots\right\} \\
+ & \left\{\frac{\partial^{2} D_{a}}{\partial \alpha^{2}} \frac{\alpha^{2}}{2 !}+\frac{\partial^{3} D_{a}}{\partial \alpha^{3}} \frac{\alpha^{3}}{3 !}+\frac{\partial^{4} D_{a}}{\partial \alpha^{4}} \frac{\alpha^{4}}{4 !}+\cdots\right\} \\
+ & \left\{\frac{\partial^{2} D_{a}}{\partial \beta^{2}} \frac{\beta^{2}}{2 !}+\frac{\partial^{3} D_{a}}{\partial \beta^{3}} \frac{\beta^{3}}{3 !}+\frac{\partial^{4} D_{a}}{\partial \beta^{4}} \frac{\beta^{4}}{4 !}+\cdots\right\} \\
+ & \left\{\frac{\partial^{2} D_{a}}{\partial P^{2}} \frac{P^{2}}{2 !}+\frac{\partial^{3} D_{a}}{\partial P^{3}} \frac{P^{3}}{3 !}+\frac{\partial^{4} D_{a}}{\partial P^{4}} \frac{P^{4}}{4 !}+\cdots\right\} \\
+ & \left\{\frac{\partial^{2} D_{a}}{\partial Q^{2}} \frac{Q^{2}}{2 !}+\frac{\partial^{3} D_{a}}{\partial Q^{3}} \frac{Q^{3}}{3 !}+\frac{\partial^{4} D_{a}}{\partial Q^{4}} \frac{Q^{4}}{4 !}+\cdots\right\} \\
& +\left\{\frac{\partial^{2} D_{a}}{\partial R^{2}} \frac{R^{2}}{2 !}+\frac{\partial^{3} D_{a}}{\partial R^{3}} \frac{R^{3}}{3 !}+\frac{\partial^{4} D_{a}}{\partial R^{4}} \frac{R^{4}}{4 !}+\cdots\right\} .
\end{aligned}
$$

Moreover the output and input of the system are $y=$ $\left[V_{T}, P, Q, R\right]^{T} u^{T}=\left[\begin{array}{llll}\delta_{T} & \delta_{e} & \delta_{a} & \delta_{r}\end{array}\right]$ where $\delta_{T}$ is the throttle position and $\delta_{e}, \delta_{a}$, and $\delta_{r}$ are the control surface positions. The following is assumed:

$$
\begin{aligned}
& \text { (A.1) }{ }^{j} \bar{\Phi} \text { is a bounded, continuous differentiable function } \\
& \text { of } x \text { in } \Omega_{j} .
\end{aligned}
$$

The assumption (A.1) implies that ${ }^{j} \bar{\Phi}$ is Lipschitz with respect to $x$ in $\Omega_{j}$. Notice that the Euler angles in (5) are related to the quaternions (2)-(3).

2.1. Problem Formulation. Let the airplane model be given by (1)-(6); find an output dependent piecewise linear control such that

$$
\lim _{t \rightarrow \infty}\left[\begin{array}{c}
V_{T} \\
P \\
Q \\
R \\
\eta \\
\epsilon
\end{array}\right]=\left[\begin{array}{c}
V_{T_{\mathrm{ref}}} \\
0 \\
0 \\
0 \\
\eta_{\mathrm{ref}} \\
\epsilon_{\mathrm{ref}}
\end{array}\right],
$$

for every $x \in D$, where $D=\cup \Omega_{j}$ and $V_{T_{\text {ref }}}$, $\eta_{\text {ref }}$, and $\epsilon_{\text {ref }}$ are the desired values of the aircraft velocity magnitude and quaternion orientation.

2.2. Control Structure. To accomplish both the movement and orientation control defined in (7), it is necessary to define the regulation error as follows:

$$
\begin{aligned}
& e_{y}=y-y_{\text {ref }}, \\
& e_{\vartheta}=\vartheta-\vartheta_{\text {ref }}
\end{aligned}
$$

Notice that (8) leads to the following implicit definition of the state error ${ }^{j} C e_{x}=e_{y}$. By substituting (8), (9) in (1) the regulation error dynamics is obtained as follows:

$$
\begin{gathered}
\dot{e}_{x}={ }^{j} A e_{x}+{ }^{j} B u+{ }^{j} \Phi_{1}\left(e_{x}\right), \\
e_{y}={ }^{j} C e_{x}, \\
\dot{e}_{9}=T\left(e_{\vartheta}\right) \omega_{\mathrm{e}},
\end{gathered}
$$

with

$$
\boldsymbol{\omega}_{\mathrm{e}} \triangleq \omega_{w}-\omega_{\mathrm{ref}}
$$

where $\omega_{w}=[P, Q, R]^{T}$ and

$$
{ }^{j} \Phi_{1}\left(e_{x}\right) \triangleq{ }^{j} \bar{\Phi}\left(e_{x}+x_{\text {ref }}\right)+{ }^{j} A x_{\text {ref }}
$$

To achieve (7) two feedback loops can be used: one that depends on the velocity regulation error (body stabilization task) and another that performs the orientation regulation. In this work the following gain scheduling control structure is studied:

$$
u=u_{1}+u_{2}
$$

where

$$
\begin{aligned}
& u_{1}=-{ }^{j} \bar{K}_{p, 1} e_{y}-{ }^{j} \varepsilon_{1}{ }^{j} K_{I, 1} \int e_{y}, \\
& u_{2}=-{ }^{j} \bar{K}_{p, 2} e_{\vartheta}-{ }^{j} \varepsilon_{2}{ }^{j} K_{I, 2} \int e_{\vartheta},
\end{aligned}
$$

with $j=1, \ldots, s$ where $s$ is the number of modes defined by the design.

${ }^{j} \bar{K}_{p, 1},{ }^{j} K_{I, 1} \in \mathbf{R}^{4 \times 4}$ are the control gains for the body stabilization loop for every mode $j$.

${ }^{j} \bar{K}_{p, 2},{ }^{j} K_{I, 2} \in \mathbf{R}^{4 \times 4}$ are the control gains for the orientation control loop for every mode $j$.

${ }^{j} \varepsilon_{1}$ and ${ }^{j} \varepsilon_{2} \in \mathbf{R}$ are constant.

The approach proposed in this work is to use the integral parameters epsilon 1 and epsilon 2 as perturbation parameters in order to establish stability conditions for the nonlinear closed-loop system. The schematic diagram of the controller (13)-(15) is given in Figure 1. To solve the regulation problem, consider the following additional assumptions:

(A.2) the triplet $\left({ }^{j} A,{ }^{j} B,{ }^{j} C\right)$ is controllable and observable for every operation mode;

(A.3) the operation domain or flight envelope $D$ satisfies $D=\cup \Omega_{j}$ with int $\Omega_{j} \bigcap$ int $\Omega_{i}=\emptyset$ for $i \neq j$ and $\Omega_{j} \neq \emptyset$.

\section{Preliminaries}

In this section, important results to deduce the main contribution of this paper are introduced. To this end, let us analyze 


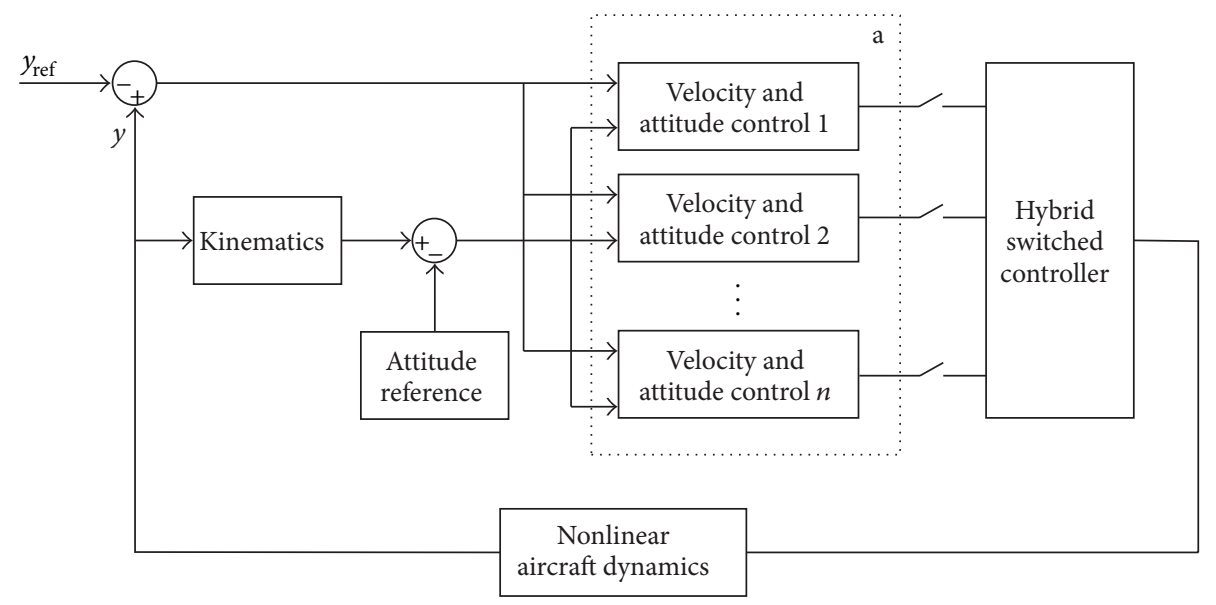

FIgURE 1: Controller structure.

the aircraft dynamics (10) under the action of the control law (14), which is given by

$$
\dot{e}=\left[\begin{array}{ccc}
{ }^{j} A_{1} & I \\
-{ }^{j} \varepsilon_{1}{ }^{j} B{ }^{j} K_{I, 1}{ }^{j} C & 0_{6 \times 6}
\end{array}\right] e+\left[\begin{array}{c}
{ }^{j} \Phi_{1}\left(e_{x}\right) \\
0
\end{array}\right],
$$

where $e_{x}, e_{s}$ are the state and quaternion tracking error, respectively, $e=\left[e_{x}, e_{s}\right]^{T} \in \mathbf{R}^{12}$ is a virtual state of the system associated with the integral action, and

$$
{ }^{j} A_{1} \triangleq{ }^{j} A-{ }^{j} B{ }^{j} \bar{K}_{p, 1}{ }^{j} C \in \mathbf{R}^{6 \times 6} .
$$

The equilibrium point of (16) is given by

$$
e_{x, \mathrm{eq}}=-{ }^{j} A_{1}^{-1}\left[b_{I}+{ }^{j} \Phi_{1}\left(e_{x, \mathrm{eq}}\right)\right] \triangleq g\left(b_{I}, e_{x, \mathrm{eq}}\right),
$$

where $\dot{e}_{s}=0 \Rightarrow e_{s}=b_{I}$ and $b_{I}$ is a constant vector. Regarding the properties of the system at equilibrium the following propositions are stated.

Proposition 1. There exists an explicit function $e_{x, e q}=h\left(b_{I}\right)$ derived from (18) if

$$
\left\|{ }^{j} A_{1}^{-1}\right\|<\frac{1}{{ }^{j} L_{d 1}},
$$

where ${ }^{j} L_{d 1}$ is the Lipschitz constant of ${ }^{j} \Phi_{1}\left(e_{x}\right)$.

Proof. Notice that

$$
\begin{aligned}
\left\|-{ }^{j} A_{1}^{-1} \nabla_{e_{x, \text { eq }}}^{j} \Phi_{1}\left(e_{x, \text { eq }}\right)\right\| & <\left\|{ }^{j} A_{1}^{-1}\right\|\left\|\nabla_{e_{x, \text { eq }}} \Phi_{1}\left(e_{x, \text { eq }}\right)\right\| \\
& <\left\|{ }^{j} A_{1}^{-1}\right\|{ }^{j} L_{d 1} .
\end{aligned}
$$

From the hypothesis, we have that $\left\|{ }^{j} A_{1}^{-1}\right\|{ }^{j} L_{d 1}<1$; therefore $\left\|-{ }^{j} A_{1}^{-1} \nabla_{e_{x, \mathrm{eq}}}{ }^{j} \Phi_{1}\left(e_{x, \mathrm{eq}}\right)\right\|<1$. Furthermore since ${ }^{j} \Phi_{1}\left(e_{x, \mathrm{eq}}\right)$ is differentiable, from (18) we have

$$
\left\|\nabla_{e_{x, \text { eq }}} g\left(e_{x, \text { eq }}\right)\right\|=\left\|-{ }^{j} A_{1}^{-1} \nabla_{e_{x, \text { eq }}}{ }^{j} \Phi_{1}\left(e_{x, \text { eq }}\right)\right\|<1 .
$$

Using the contraction mapping theorem [24], there exists a unique solution of (18); hence there exists an explicit map $e_{x, \mathrm{eq}}=h\left(b_{I}\right)$.

In other words, if ${ }^{j} \bar{K}_{p, 1} \triangleq{ }^{j} \gamma^{j} K_{p, 1}$, where ${ }^{j} \gamma>0$, hence (20) can be rewritten as

$$
\begin{gathered}
\left\|{ }^{j} \gamma\left(\frac{{ }^{j} A}{{ }^{j} \gamma}-{ }^{j} B{ }^{j} K_{p, 1}{ }^{j} C\right)\right\| \leq\left|{ }^{j} \gamma\right|\left\|\left(\frac{{ }^{j} A}{{ }^{j} \gamma}-{ }^{j} B{ }^{j} K_{p, 1}{ }^{j} C\right)\right\|, \\
{ }^{j} L_{d 1}<{ }^{j} \gamma\left\|\frac{{ }^{j} \gamma}{{ }^{j} \gamma}-{ }^{j} B{ }^{j} K_{p, 1}{ }^{j} C\right\| .
\end{gathered}
$$

From (22) it can be seen that if ${ }^{j} \gamma \rightarrow \infty$, then ${ }^{j} \gamma\left\|\left({ }^{j} A /{ }^{j} \gamma\right)-{ }^{j} B{ }^{j} K_{p, 1}{ }^{j} C\right\| \rightarrow \infty$. Such results imply the existence of a sufficiently large ${ }^{j} \gamma$ such that condition of this proposition is satisfied.

Proposition 2. Considering the map

$$
e_{s}=-{ }^{j} A_{1} e_{x}-{ }^{j} \Phi_{1}\left(e_{x}\right),
$$

there exist real negative eigenvalues of ${ }^{j} A_{1}$, such that $\left[\nabla_{e_{x}} e_{s}\right]^{-1}$ is positive definite.

Proof. Computing the $e_{x}$-derivative of (23), we have

$$
\nabla_{e_{x}} e_{s}=-{ }^{j} A_{1}-\nabla_{e_{x}}{ }^{j} \Phi_{1}\left(e_{x}\right) .
$$

According to Theorem 3 in [25] and Assumption (A.2), the eigenvalues of (17) can be arbitrarily assigned (by virtue of gain ${ }^{j} \bar{K}_{p, 1}$ ). Therefore, they are chosen for ${ }^{j} A_{1}$ to be negative definite. Notice that in view of this fact ${ }^{j} A_{1}$ is Hurwitz and ${ }^{-}{ }^{j} A_{1}$ is positive definite. Moreover, given (17), if ${ }^{j} \bar{K}_{p, 1}=$ ${ }^{j} \gamma{ }^{j} K_{p, 1}$, there exists a sufficiently large value of ${ }^{j} \gamma$ such that the term ${ }^{j} A_{1}$ prevails over $\nabla_{e_{x}}{ }^{j} \Phi_{1}\left(e_{x}\right)$, which by virtue of Assumption (A.1) is bounded in $\Omega_{j}$. These arguments lead 
us to deduce the existence of controller gains ${ }^{j} \bar{K}_{p, 1}$ such that $\nabla_{e_{x}} e_{s}$ is positive definite, which implies that $\left[\nabla_{e_{x}} e_{s}\right]^{-1}$ is positive definite.

\section{Main Results}

In this section, the main contributions of this work are stated in Theorems 3 and 4 . These theorems contain results regarding the stability of every operation mode (i.e., stability of a two-loop controller acting in a given operation region) and the stability of the entire operation region or flight envelope conditions.

4.1. Stability of Every Operation Mode. This stability analysis is performed in two parts; firstly the stability of the body stabilization loop is analyzed and afterwards the orientation stabilization loop. The stability analysis makes use of singularly perturbed systems [26], since the idea is to use integral control gains as perturbation parameters. In other words, since the integral actions constitute slow actions of the controller, the closed-loop system can be described naturally using two systems: a fast system given by the aircraft dynamics under a pure proportional action and a slow system given by the integral dynamics of the regulation error. The singular perturbed system arises as the integral gains approach zero. The interested reader is referenced to [26, 27] for a more detailed explanation of singularly perturbed systems.

Theorem 3. Let the dynamics of the aircraft be described by (10)-(15) in the set $\Omega_{j}$, let ${ }^{j} K_{I, i} \in \mathbf{R}^{4 \times 4}, i \in\{1,2\}, j \in\{1, \ldots, s\}$, and let ${ }^{j} \bar{K}_{p, 1}$ be as in Propositions 1 and 2; then there exist constants $0<{ }^{j} \varepsilon_{1}<\varepsilon_{\max }$ and $0<{ }^{j} \varepsilon_{2}<\varepsilon_{\max }$, such that the origin of (10) under the action of control (13) is asymptotically stable.

Proof. As a first step, consider the aircraft under the body stabilization loop. From Proposition 1, a unique isolated equilibrium exists; let us denote such equilibrium values as $e_{x, \text { eq }}$ and $e_{s, \mathrm{eq}}$. In view of this fact, the following coordinates are introduced to shift such equilibrium to the origin

$$
\begin{aligned}
& z \triangleq e_{x}-e_{x, \mathrm{eq}}, \\
& w \triangleq e_{s}-e_{s, \mathrm{eq}} .
\end{aligned}
$$

At this point, let us use variable ${ }^{j} \varepsilon_{1}>0$ as a perturbation parameter; hence the closed-loop system (10)-(13) can be rewritten as a standard singularly perturbed system given by

$$
\left[\begin{array}{c}
{ }^{j} \varepsilon_{1} z^{\prime} \\
w^{\prime}
\end{array}\right]=\left[\begin{array}{ccc}
{ }^{j} A_{1} & & I \\
-{ }^{j} B{ }^{j} K_{I, 1}{ }^{j} C & 0_{4 \times 4}
\end{array}\right]\left[\begin{array}{c}
z \\
w
\end{array}\right]+\left[\begin{array}{c}
{ }^{j} \Phi_{2}(z) \\
0
\end{array}\right],
$$

where ${ }^{j} \Phi_{2}(z) \triangleq j \Phi_{1}\left(z+e_{x, \mathrm{eq}}\right), t^{\prime}={ }^{j} \epsilon_{1} t, z \triangleq z\left(t^{\prime}\right), z^{\prime} \triangleq$ $d z / d t^{\prime}, w \triangleq w\left(t^{\prime}\right)$, and $w^{\prime} \triangleq d w / d t^{\prime}$.
The boundary-layer and reduced systems associated with (26) are given, respectively, by

$$
\begin{gathered}
\dot{z}={ }^{j} A_{1} z+{ }^{j} \Phi_{2}(z), \\
0={ }^{j} A_{1} z+w+{ }^{j} \Phi_{2}(z), \\
w^{\prime}=-{ }^{j} B{ }^{j} K_{I, 1}{ }^{j} C z .
\end{gathered}
$$

Observe that (27) can be seen as the aircraft system under pure proportional action. On the other hand, notice that (28) is a steady-state relationship between $z$ and $w$ that satisfies $z=-{ }^{j} A_{1}^{-1}\left[w+{ }^{j} \Phi_{2}(z)\right]$, which has the same structure of (18); therefore Proposition 1 can be used; hence, for a suitable proportional gain (i.e., sufficiently large ${ }^{j} \gamma$ ), there exists an explicit function $z \triangleq h(w)$ such that

$$
w^{\prime}=-{ }^{j} B^{j} K_{I, 1}{ }^{j} C h(w) .
$$

Firstly, we will show that the linear output feedback (14) is able to track asymptotically the velocity of the nonlinear aircraft. Observe that (30) can be seen as the aircraft system under pure integral action. To derive the stability properties of (26), we can use systems (27) and (30).

We start by showing that, for a sufficiently large ${ }^{j} \gamma$, the origin of (27) is exponentially stable. To this end, let us consider the following scalar function:

$$
{ }^{j} V_{1}=z^{T j} P_{1} z
$$

where ${ }^{j} P_{1} \in \mathbf{R}^{6 \times 6}$ is positive definite. Since ${ }^{j} A_{1}$ is Hurwitz, the time derivative of (31) along (27) is given by

$$
{ }^{j} \dot{V}_{1}=-z^{T}{ }^{j} Q_{1} z+2 z^{T}{ }^{j} P_{1}{ }^{j} \Phi_{2}(z),
$$

with ${ }^{j} Q_{1}$ positive definite, since ${ }^{j} \Phi_{2}(z)$ is Lipschitz; hence

$$
\begin{gathered}
{ }^{j} \dot{V}_{1} \leq-\|z\|^{2}\left\|{ }^{j} Q_{1}\right\|+2\|z\|^{2}\left\|{ }^{j} P_{1}\right\| L_{1}, \\
{ }^{j} \dot{V}_{1} \leq\left(-\left\|{ }^{j} Q_{1}\right\|+2 L_{1}\left\|{ }^{j} P_{1}\right\|\right)\|z\|^{2},
\end{gathered}
$$

where $L_{1}$ is the Lipschitz constant of ${ }^{j} \Phi_{2}(z)$. To ensure that $\left(-\left\|{ }^{j} Q_{1}\right\|+2 L_{1}\left\|{ }^{j} P_{1}\right\|\right)$ is negative definite, it is required that $2 L_{1}\left\|{ }^{j} P_{1}\right\| \leq\left\|{ }^{j} Q_{1}\right\|$, since $\left\|{ }^{j} Q_{1}\right\|=\left\|{ }^{j} A_{1}^{T}{ }^{j} P_{1}+{ }^{j} P_{1}{ }^{j} A_{1}\right\|$ and ${ }^{j} \bar{K}_{p, 1}={ }^{j} \gamma^{j} K_{p, 1}$ with ${ }^{j} \gamma>0$; hence

$$
\begin{aligned}
\left\|Q_{1}\right\|= & \left\|{ }^{j} A_{1}^{T}{ }^{j} P_{1}+{ }^{j} P_{1}{ }^{j} A_{1}\right\| \\
= & \|{ }^{j} \gamma\left[\frac{{ }^{j} A}{{ }^{j} \gamma}-{ }^{j} B{ }^{j} K_{p, 1}{ }^{j} C\right]^{T} P_{1} \\
& +{ }^{j} \gamma{ }^{j} P_{1}\left[\frac{{ }^{j} A}{{ }^{j} \gamma}-{ }^{j} B{ }^{j} K_{p, 1}{ }^{j} C\right] \| \\
= & \left\|{ }^{j} \gamma\right\| \|\left[\frac{{ }^{j} A}{{ }^{j} \gamma}-{ }^{j} B{ }^{j} K_{p, 1}{ }^{j} C\right]{ }^{j} P_{1} \\
& +{ }^{j} P_{1}\left[\frac{{ }^{j} A}{{ }^{j} \gamma}-{ }^{j} B{ }^{j} K_{p, 1}{ }^{j} C\right] \| .
\end{aligned}
$$


From (34) it can be observed that $\lim _{j} \gamma_{\rightarrow \infty}\left\|{ }^{j} Q_{1}\right\|=\infty$, which implies that, for a sufficiently large ${ }^{j} \gamma$, the origin of (27) is exponentially stable.

On the other hand, to study stability of the reduced system (30), consider the scalar function ${ }^{j} V_{2}=(1 / 2) w^{T} w$. Let $E \triangleq$ ${ }^{j} B{ }^{j} K_{I, 1}{ }^{j} C$; hence the time derivative of ${ }^{j} V_{2}$ along (27) is ${ }^{j} \dot{V}_{2}=-w^{T} E h(w)$.

Using the result in Proposition 2, $\nabla_{w} h(w)=\left[\nabla_{e_{x}} e_{s}\right]^{-1}$ is positive definite, which in turn implies that ${ }^{j} \dot{V}_{2}$ is positive semidefinite. Using the Lasalle invariance principle and provided that ${ }^{j} \dot{V}_{2}=0$ only in $z=0, w=0$, it is possible to conclude that the origin of (27) is asymptotically stable.

At this point, asymptotic stability of (16) can be concluded using Theorem 2.1 in [26]. The stability properties of the reduced and boundary layer system imply the existence of a parameter $\varepsilon_{\max }>0$ such that, for every $0<{ }^{j} \varepsilon_{1}<\varepsilon_{\max }$, the origin of (16) is asymptotically stable, with a scalar function

$$
{ }^{j} V_{3}=\frac{\left({ }^{j} V_{1}+{ }^{j} V_{2}\right)}{{ }^{j} \varepsilon_{1}} .
$$

So far, it has been shown that the linear output feedback (14) is able to asymptotically stabilize the velocity of the nonlinear aircraft.

To show that the second linear loop (15) is able to regulate the orientation, consider the following: let us compute the closed-loop system (10)-(15) under steady-state conditions:

$$
\begin{gathered}
0=f\left(\sigma_{\mathrm{eq}}\right)-\Lambda\left(e_{\vartheta, \mathrm{eq}} ;{ }^{j} K_{p, 2}\right)+\bar{B}\left(\rho_{\mathrm{eq}}\right), \\
0=T\left(e_{\vartheta, \mathrm{eq}}\right)[P, Q, R]^{T}, \\
0={ }^{j} \varepsilon_{2}{ }^{j} K_{I, 2} e_{9, \mathrm{eq}},
\end{gathered}
$$

where the subindex eq is the value of the variable under equilibrium conditions, $\sigma=[z, w]^{T}, f(\sigma)$ is the right-hand side of (26), $\rho$ is the virtual state associated with the integral action, $\bar{B}(\rho) \triangleq\left[{ }^{j} B \rho, 0\right]^{T}$, and

$$
\Lambda\left(e ;{ }^{j} K_{p, 2}\right) \triangleq\left[\begin{array}{c}
{ }^{j} B{ }^{j} K_{p, 2} e_{\vartheta} \\
0
\end{array}\right] .
$$

From (38) it is clear that $e_{9, \mathrm{eq}}=0$, since ${ }^{j} K_{I, 2}$ is full rank. Observe that $T(0)=0, \Lambda\left(0 ;{ }^{j} K_{p, 2}\right)=0$, and $\bar{B}\left(\rho_{\mathrm{eq}}\right)$ is a constant vector, so (36) can be rewritten as

$$
0=f\left(\sigma_{\mathrm{eq}}\right)+\bar{B}\left(\rho_{\mathrm{eq}}\right)
$$

Observe that (40) has the same structure as (18), except for the translation term $\bar{B}\left(\rho_{\mathrm{eq}}\right)$. Hence Proposition 1 can be used to guarantee the existence and uniqueness of the equilibrium point. That implies that in steady-state conditions for every value of the state one and only one integral action corresponds. As before, let us consider system (26) under the action of controller (15) as a singularly perturbed system, with the gain of the integral action of the second loop as a new perturbation parameter. In this way, such closed-loop system can be rewritten as:

$$
\begin{gathered}
{ }^{j} \mathcal{E}_{2} \widetilde{\sigma}^{\prime}=f\left(\widetilde{\sigma}^{\prime}\right)-\Lambda\left(\widetilde{e}_{9} ;{ }^{j} K_{p, 2}\right)+\bar{B}\left(\widetilde{\rho}^{\prime}\right), \\
{ }^{j} \varepsilon_{2} \widetilde{e}_{\vartheta}^{\prime}=T\left(\vec{e}_{\vartheta}^{\prime}\right)[P, Q, R]^{T}, \\
\widetilde{\rho}^{\prime}=-{ }^{j} K_{I, 2} \vec{e}_{\vartheta}^{\prime},
\end{gathered}
$$

with $\widetilde{\sigma}=[\widetilde{z}, \widetilde{w}] \triangleq \sigma-\sigma_{\text {eq }}=\left[z-z_{\text {eq }}, w-w_{\text {eq }}\right], \widetilde{\rho} \triangleq \rho-\rho_{\text {eq }}$, $\tau^{\prime}={ }^{j} \epsilon_{2} t$ is the new time scale, and $\widetilde{e}_{9} \triangleq e_{9}-e_{9, \text { eq }}, \widetilde{\sigma}^{\prime} \triangleq$ $d \widetilde{\sigma} / d \tau^{\prime}, \widetilde{\rho}^{\prime} \triangleq d \widetilde{\rho} / d \tau^{\prime}$, and $\widetilde{e}_{9}^{\prime} \triangleq d \widetilde{e}_{9} / d \tau^{\prime}$. Observe that (41) has an equilibrium point in $\left(\widetilde{\sigma}, \widetilde{e}_{9}, \widetilde{\rho}\right)=(0,0,0)$. Moreover, observe that $\left(\widetilde{\sigma}, \widetilde{e}_{\vartheta}, \widetilde{\rho}\right) \rightarrow(0,0,0)$, as $\tau^{\prime} \rightarrow \infty$ implies that $\left(\widetilde{\sigma}, \widetilde{e_{9}}, \widetilde{\rho}\right) \rightarrow(0,0,0)$ as $t \rightarrow \infty$.

At this point, let us compute the reduced and boundary layer systems associated with (41), which are given, respectively, by

$$
\begin{gathered}
\widetilde{\rho}^{\prime}=-{ }^{j} K_{I, 2}{ }^{j} K_{p, 2}^{-1} \widetilde{\rho}, \\
\dot{\widetilde{\sigma}}=f(\widetilde{\sigma}) .
\end{gathered}
$$

Notice that (43) has been studied before; the origin of such system is asymptotically stable for $0<{ }^{j} \varepsilon_{1}<\varepsilon_{\max }$. On the other hand, the stability of the reduced system (42) can be readily established by suitable gains $-{ }^{j} K_{I, 2}^{j} K_{p, 2}$ that make the matrix Hurwitz, hence, the existence of a Lyapunov function ${ }^{j} V_{4}=\tilde{\rho}^{T j} P_{4} \tilde{\rho}$ associated with (42) can be derived such that ${ }^{j} \dot{V}_{4}=-\widetilde{\rho}^{T j} Q_{4} \tilde{\rho}<0$.

Given the arguments above and the Lipschitz nature of ${ }^{j} \Phi_{2}(\widetilde{z})$, Theorem 2.1 in [26] implies the existence of a parameter $\varepsilon_{\max }>0$ such that for all $0<{ }^{j} \varepsilon_{2}<\varepsilon_{\max }$ the origin of (41) is asymptotically stable.

So far, it has been proven that a linear control is able to stabilize both velocity and orientation of the nonlinear aircraft; however, such result has been derived from the hypothesis that the system description is continuous. To relax such assumption, in the following we will study the stability of a family of piecewise continuous functions, which describes the dynamic of the aircraft at a family of desired operation points.

4.2. Stability of the Switching Structure. Conditions in Theorem 3 guarantee the stability in a local domain $\Omega_{j}$. However, they do not constitute sufficient evidence to guarantee the stability of the entire flight envelope. To obtain such evidence, the piecewise continuous system under the action of discrete event switching has to be studied.

Since the origin of every continuous description of the system can be defined using different operation points (reference), the switching among modes can be described using discrete event switching depending on time, system's state, or both.

In view of this fact, it is necessary to study the requirements on the discrete event actions that ensure asymptotic stability of the closed-loop hybrid system. The following theorem establishes such conditions. 
Theorem 4. Assume that conditions of Theorem 3 are satisfied and let

$$
{ }^{j} V_{\text {tot }}(\pi)=z^{T j} P_{1} z+\frac{1}{2} w^{T} w+\widetilde{\rho}^{T j} P_{4} \widetilde{\rho} \triangleq \pi^{T}{ }^{j} \pi,
$$

with $\pi^{T}=[z, w, \tilde{\rho}]$. The origin of the hybrid system (10)(13) is asymptotically stable for the entire flight envelope using switching criteria guaranteeing an average dwell time for the $j$ th mode, $T_{A D}>0$, such that

$$
{ }^{j} V_{\text {tot }}\left(\pi\left({ }^{j+} t_{l}\right)\right) \geq{ }^{j} V_{\text {tot }}\left(\pi\left({ }^{j-} t_{l+1}\right)\right),
$$

with ${ }^{j+} t_{l},{ }^{j-} t_{l}$ are the initial time and final time when the jth mode becomes active for the lth time, respectively.

Proof. The proof will be performed using Theorem 7 in [28]. According to the result in [28], the existence of a differentiable and radially unbounded scalar function ${ }^{j} V_{\text {tot }}>0$ must be shown such that ${ }^{j} \dot{V}_{\text {tot }} \leq 0$ along trajectories of (41). Let us consider the following quadratic positive definite scalar function:

$$
{ }^{j} V_{\text {tot }}(\pi)=z^{T j} P_{1} z+\frac{1}{2} w^{T} w+\widetilde{\rho}^{T j} P_{4} \tilde{\rho} .
$$

From Theorem 3,

$$
\alpha_{1}(\|\pi\|) \leq{ }^{j} V_{\text {tot }}(\pi) \leq \alpha_{2}(\|\pi\|),
$$

with $\alpha_{2}(\|\pi\|)=3 \max _{j}\left\{\lambda_{\max }\left({ }^{j} P_{1}\right) /{ }^{j} \varepsilon_{1}{ }^{j} \varepsilon_{2}, 1 /{ }^{j} \varepsilon_{1}{ }^{j} \varepsilon_{2}\right.$, $\left.\lambda_{\text {max }}\left({ }^{j} P_{4}\right) /{ }^{j} \varepsilon_{2}\right\}\|\pi\|^{2}$, and $\alpha_{1}(\|\pi\|)=3 \min _{j}\left\{\lambda_{\text {min }}\left({ }^{j} P_{1}\right) /\right.$ $\left.{ }^{j} \varepsilon_{1}{ }^{j} \varepsilon_{2}, 1 /{ }^{j} \varepsilon_{1}{ }^{j} \varepsilon_{2}, \lambda_{\text {min }}\left({ }^{j} P_{4}\right) /{ }^{j} \varepsilon_{2}\right\}\|\pi\|^{2}$. On the other hand, the time derivative of (44) along (10)-(13) is given by

$$
\begin{aligned}
& { }^{j} \dot{V}_{\text {tot }}(\pi) \\
& \leq-\left\{\left\{-\left\|{ }^{j} Q_{1}\right\|+2 L_{1}\left\|{ }^{j} P_{1}\right\|\right\}\|z\|^{2}+w^{T} E h(w)+\tilde{\rho}^{T j} Q_{4} \tilde{\rho}\right\} \\
& \triangleq-{ }^{j} W_{\text {tot }}(\pi) .
\end{aligned}
$$

From Theorem 3 in this work, the origin of every operation mode $j$ is asymptotically stable. Using this property and Remark 6 in [28] it is possible to conclude the satisfaction of Conditions 1 and 2 of Theorem 7 in [28], that is, the existence of a class $\mathscr{K}_{\infty}$ function $(\kappa)$ such that $|\pi(t)| \leq$ $\kappa\left(\left\|{ }^{j} W_{\text {tot }}(\pi)\right\|_{[t, t+\tau]}\right)$.

Finally, Remark 7 and Lemma 8 in [28] let us conclude that if a minimum average dwell time $T_{\mathrm{AD}}>0$,

$$
{ }^{j} V_{\text {tot }}\left(\pi\left({ }^{j+} t_{l}\right)\right) \geq{ }^{j} V_{\text {tot }}\left(\pi\left({ }^{j-} t_{l+1}\right)\right),
$$

with ${ }^{j-} t_{l},{ }^{j+} t_{l}$ are the initial time and final time when the $j$ th mode becomes active for the $l$ th time, respectively, the origin of the hybrid system is asymptotically stable in $D$, where $D=$ $\cup \Omega_{j}$.
Remark 5. Theorem 4 provide us with guidelines to design switching laws for the hybrid system. According to such theorem, the gain scheduling control must use a supervisory action that decides whether a given mode is active using switching laws that can be either time or state dependent, as long as a minimum average dwell time is satisfied for every mode. This average time can be arbitrary small as long as $T_{\mathrm{AD}}>0$. The stability result in Theorem 4 actually establishes that the system will be stable if chattering does not occur. The phenomenon of chattering occurs when infinite switching actions occur in finite time and it may lead to finite time destabilization.

Remark 6. The results discussed in Theorems 3 and 4 are in fact quite general, and can be used to other systems that admit the same description, including aircrafts with some actuator failures. That is, the same result can be applied to other highly nonlinear systems as long as Assumptions (A.1)-(A.3) are satisfied and the piecewise description of the system fits the model in (10)-(13).

\section{Illustrative Results}

The aim of this section is to illustrate the theoretical results above. To this end, the nonlinear model of F16 (see [29]) is simulated using Simulink. In order to illustrate the effect of the uncertainty, the simulations are conducted as follows: firstly, a set of linear approximations are proposed at different operation points, each of these approximations is associated with a domain $\Omega_{j}$ and the linear approximation is used only to perform the controller tuning at the corresponding modes. Secondly, the gains are used to control a piecewise nonlinear model of the system whose modes coincide with the modes of the linear counterpart. For illustration purposes only two flight modes are used, namely, steady-state fight at different cruise velocities.

Firstly, the effect of the integral action is evaluated tracking a sinusoidal function. The switching criterion is chosen to be velocity dependent and at the surface $v=325 \mathrm{ft} / \mathrm{s}$, so to perform the task the aircraft must switch consecutively between Mode 1 and Mode 2. The results are displayed in Figures 2, 3, and 4 .

Figure 2 shows the time evolution of the cruise velocity for three different integral gains, which, according to Theorem 3, are parameterized using ${ }^{j} \varepsilon_{1}$. Figure 2 shows that for simplicity ${ }^{1} \varepsilon_{1}={ }^{2} \varepsilon_{1}=\varepsilon_{1}$ have been chosen and the three integral parameters are denoted as $\varepsilon_{1, a}, \varepsilon_{1, b}, \varepsilon_{1, c}$. Theorem 3 establishes the existence of a maximum integral gain such that the system is asymptotically stable. For $\varepsilon_{1, a}<\varepsilon_{1, b}$, the velocity reference can be followed; however, for $\varepsilon_{1, c} \gg \varepsilon_{1, b}$, the system leads to instability.

In Figure 3 it can be observed that for $\varepsilon_{1, a}$ the body stabilization task is able to avoid rotation, still with a short transient period. Such transient response is reduced with $\varepsilon_{1, b}$ and finally with $\varepsilon_{1, c}$ the aircraft is unstable. That is, moderate values of the integral action result in a successful velocity and orientation tracking and good performance. However since the maximum value of the integral gain that can be 


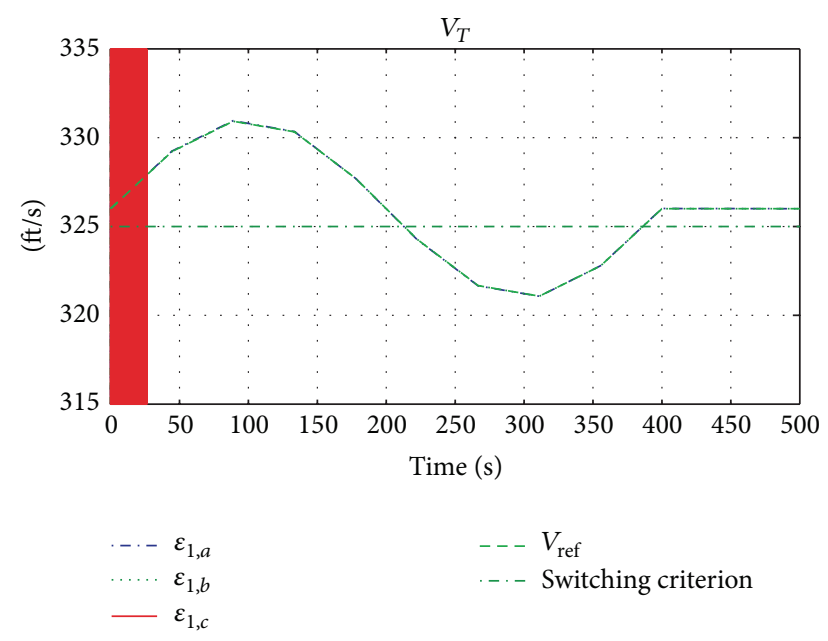

FIGURE 2: Time evolution of aircraft cruise velocity for three different integral gains $\left(\varepsilon_{1, a}>\varepsilon_{1, b}>\varepsilon_{1, c}\right)$.
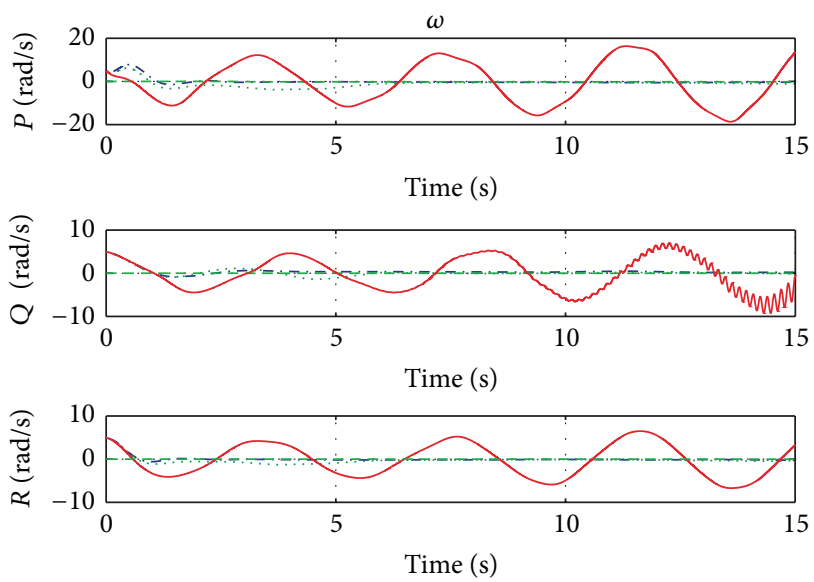

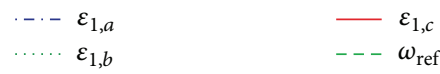

FIGURE 3: Time evolution of the aircraft attitude for three different integral gains $\left(\varepsilon_{1, a}>\varepsilon_{1, b}>\varepsilon_{1, c}\right)$.

used is upper bounded, a performance criterion to choose the integral gains can be used to define properly the operation domains $\Omega_{j}$.

Similar observations can be performed for the orientation loop (see Figure 4), which integral gains can be parameterized using ${ }^{j} \mathcal{E}_{2}$. For simplicity, the integral parameters are chosen such that ${ }^{1} \varepsilon_{2}={ }^{2} \varepsilon_{2}=\varepsilon_{2}$. Three different gains are evaluated $\varepsilon_{2, a}>\varepsilon_{2, b}>\varepsilon_{2, c}$. For small integral gains, the orientation can be successfully tracked $\left(\varepsilon_{2, a}\right)$; however, higher gains lead firstly to a performance degradation $\left(\varepsilon_{2, b}\right)$ and secondly to instability $\left(\varepsilon_{2, c}\right)$.

Finally, to illustrate the result in Theorem 4 , fast switching actions are induced by following a high frequency sinusoidal velocity reference (see Figures 5 and 6). The switching conditions are velocity dependent and set at a cruise velocity of
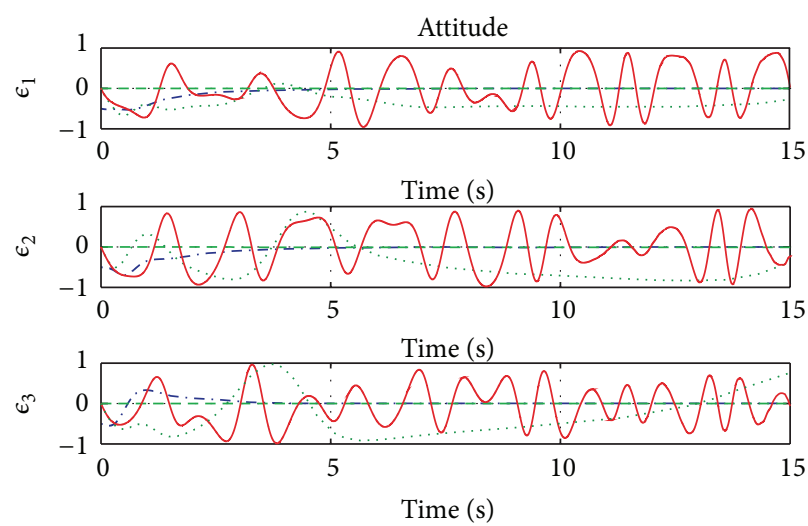

$$
\begin{array}{ll}
-\cdot-\varepsilon_{2, a} & -\varepsilon_{2, c} \\
\cdots-\varepsilon_{2, b} & ---\varepsilon_{\text {ref }}
\end{array}
$$

FIgURE 4: Time evolution of the angular velocity for three different integral gains $\left(\varepsilon_{2, a}>\varepsilon_{2, b}>\varepsilon_{2, c}\right)$.

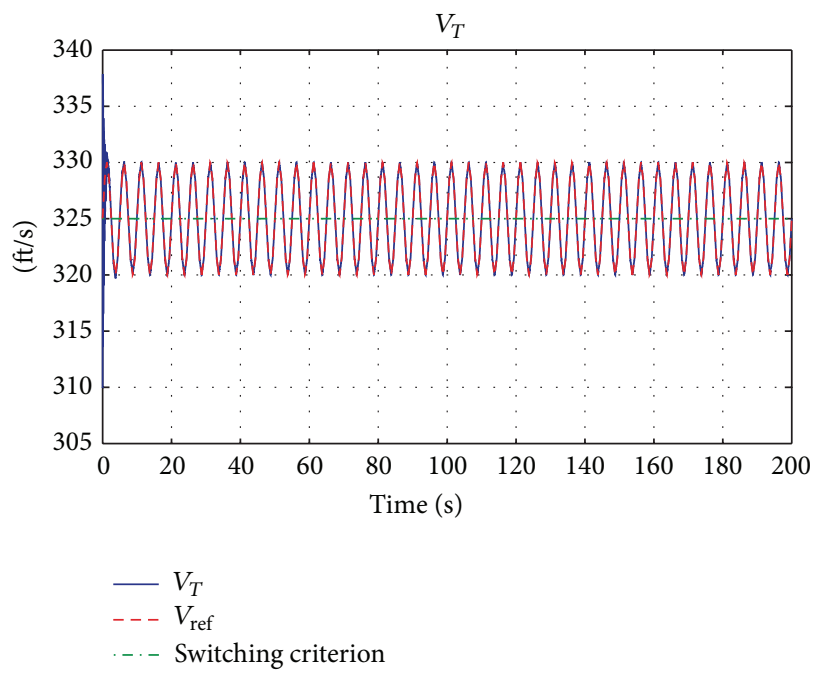

FIGURE 5: Time evolution of aircraft cruise velocity under fast switching conditions.

$35 \mathrm{ft} / \mathrm{sec}$. In Figure 6 it is possible to observe the corresponding behavior of the angular velocity and the aircraft attitude. Notice that even if the model is switching continuously, the evolution of the system remains stable and, for the controller gains in the simulations, they are able to track correctly the desired reference. Such result agrees with theoretical results in Theorem 4.

\section{Conclusions}

In this work, the stability properties of an aircraft with nonlinear behavior controlled with a gain scheduled approach are studied. It is shown that the inclusion of a linear integral action to an output feedback linear controller makes it possible to guarantee asymptotical tracking of velocity and orientation in spite of nonlinearities and uncertainties. This result is interesting since even if the behavior of the aircraft 

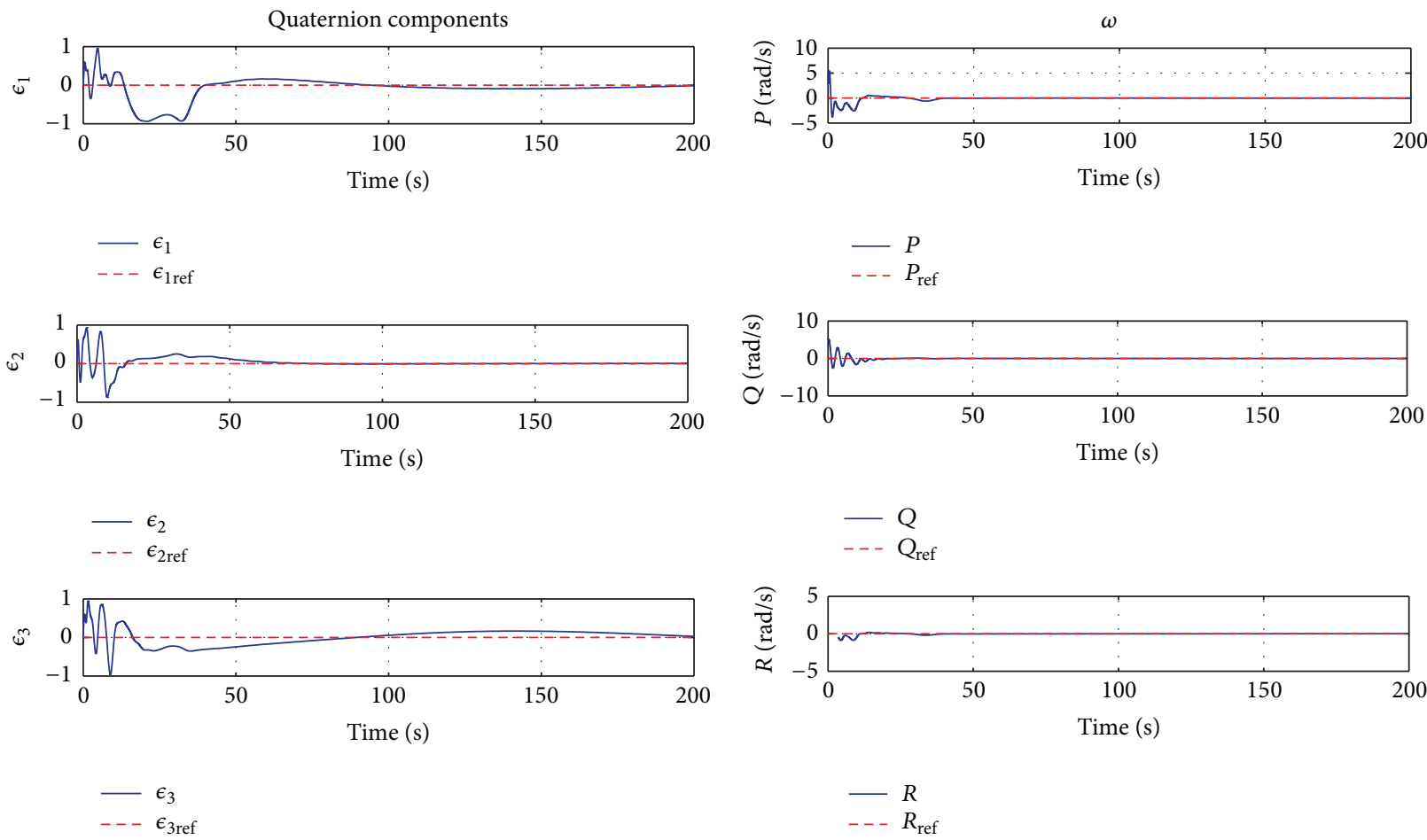

(a)

(b)

FIGURE 6: Time evolution of (a) aircraft attitude and (b) angular velocity under fast switching conditions.

system is highly nonlinear, the controller gains can be tuned using linear approximations of the system in given operation points and the performance of the system can be set using a trade-off between the size of the integral gains and the size of the operating domain $\Omega_{j}$. The switching criteria can be either time or state dependent as long as a minimum residence time $\left(T_{\mathrm{AD}}>0\right)$ is used in every mode and it can be designed using a supervisory structure which evaluates the satisfaction of condition (45). Due to this fact the control scheme is flexible and physical or user-defined restrictions can be taken into account.

\section{Nomenclature}

$\begin{array}{ll}\alpha: & \text { Angle of attack } \\ \alpha_{e}: & \text { Angle of attach at the operation point } \\ \alpha_{T}: & \text { Drift angle } \\ \beta: & \text { Side slip angle } \\ { }^{j} \gamma: & \text { Scalar associated with the } \\ & \text { proportional gain of the first loop } \\ \gamma_{e}: & \text { Flight path angle } \\ \Gamma: & J_{Z}^{\prime} J_{X}^{\prime}-J_{X Z}^{\prime} \\ \delta_{T}: & \text { Throttle position } \\ \delta_{e}, \delta_{a}, \text { and } \delta_{r}: & \text { Control surface positions (elevator, } \\ & \text { aileron, and rudder) } \\ { }^{j} \varepsilon_{1}: & \text { Scalar associated with the integral } \\ { }^{j} \varepsilon_{\varepsilon_{2}}: & \text { gain of the first loop } \\ & \text { Scalar associated with the integral } \\ & \text { gain of the second loop }\end{array}$

$\begin{array}{ll}\eta, \epsilon_{1}, \epsilon_{2}, \epsilon_{3}: & \text { Quaternion components } \\ \mu: & J_{Z}^{\prime} J_{X}^{\prime} / \Gamma \\ \sigma_{1}: & J_{Z}^{\prime} J_{X Z}^{\prime} / \Gamma \\ \sigma_{2}: & J_{X}^{\prime} J_{X Z}^{\prime} / \Gamma \\ \phi, \theta, \psi: & \text { Euler angles } \\ { }^{j} \bar{\Phi}(x): & \text { Nonlinearities vector } \\ \Omega_{j}: & \text { Mode domain } \\ { }^{j} A: & \text { State matrix for mode } j \\ { }^{j} B: & \text { Control matrix for mode } j \\ C: & \text { Output matrix } \\ D_{D}: & \text { Flight envelope domain } \\ D_{a}, L, C_{a}: & \text { Drag, lift, and crosswind forces } \\ D_{a e}, L_{e}, C_{a e}: & \text { Drag, lift, and crosswind forces at the } \\ F_{T}: & \text { operation point } \\ g: & \text { Traction force } \\ I_{i}: & \text { Gravity acceleration } \\ i: & \text { Identity matrix } \\ I_{x}^{\prime}, I_{y}^{\prime}, I_{z}^{\prime}, I_{x z}^{\prime}: & \text { Loop index } \\ j: & \text { Inertia matrix components (body } \\ J_{w}, J_{X}^{\prime}, J_{Y}^{\prime}, J_{Z}^{\prime}, J_{X Z}^{\prime}: & \text { Irame) } \\ l_{w}, m_{w}, n_{w}: & \text { Waypoint or mode index } \\ l_{w_{e}}, m_{w_{e}}, n_{w_{e}}: & \text { frame) } \\ L_{\beta}: & \text { Rolling, pitching, and yawing } \\ & \text { moment } \\ & \text { moment at the operation point } \\ & \left.\text { (1/ J J } J_{X}^{\prime}\right)\left(\partial l_{w} / \partial \beta \text { ) }\right. \\ & \end{array}$




\begin{tabular}{|c|c|}
\hline$L_{p}:$ & $\left(1 / J_{X}^{\prime}\right)\left(\partial l_{w} / \partial P\right)$ \\
\hline$L_{r}^{P}:$ & $\left(1 / J_{X}^{\prime}\right)\left(\partial l_{w} / \partial R\right)$ \\
\hline $\mathrm{m}:$ & Aircraft mass \\
\hline$M_{\alpha}:$ & $\left(1 / J_{Y}^{\prime}\right)\left(\partial m_{w} / \partial \alpha\right)$ \\
\hline$M_{q}:$ & $\left(1 / J_{Y}^{\prime}\right)\left(\partial m_{w} / \partial Q\right)$ \\
\hline$m_{w}:$ & Trust moment (body frame) \\
\hline$M_{T_{\alpha}}:$ & $\left(1 / J_{Y}^{\prime}\right)\left(\partial m_{T} / \partial \alpha\right)$ \\
\hline$M_{V}^{\alpha}$ & $\left(1 / J_{Y}^{\prime}\right)\left(\partial m_{w} / \partial V_{T}\right)$ \\
\hline$M_{T_{V}}:$ & $\left(1 / J_{Y}^{\prime}\right)\left(\partial m_{T} / \partial V_{T}\right)$ \\
\hline$N_{\beta}:$ & $\left(1 / J_{Z}^{\prime}\right)\left(\partial n_{w} / \partial \beta\right)$ \\
\hline$N_{p}^{P}:$ & $\left(1 / J_{Z}^{\prime}\right)\left(\partial n_{w} / \partial P\right)$ \\
\hline$N_{r}^{P}:$ & $\left(1 / J_{Z}^{\prime}\right)\left(\partial n_{w} / \partial R\right)$ \\
\hline
\end{tabular}

$P, Q, R$ : Angular velocity components (roll, pitch, and yaw)

$u$ : Controlled input vector

$V_{T}$ : Magnitude of airplane linear velocity

$V_{T_{e}}$ : Airplane linear velocity at the operation point

$x: \quad$ State vector

$X_{\alpha}: \quad(1 / \mathrm{m})\left[L-\left(\partial D_{a} / \partial \alpha\right)\right]$

$X_{V}: \quad(-1 / \mathrm{m})\left(\partial D_{a} / \partial V_{T}\right)$

$X_{T_{V}}: \quad(1 / \mathrm{m})\left(\partial F_{T} / \partial V_{T}\right)$

$y: \quad$ Output vector

$Y_{\beta}: \quad(1 / \mathrm{m})\left[-D_{a}+\left(\partial C_{a} / \partial \beta\right)\right]$

$Y_{p}: \quad(1 / \mathrm{m})\left(\partial C_{a} / \partial P\right)$

$Y_{r}: \quad(1 / \mathrm{m})\left(\partial C_{a} / \partial R\right)$

$Z_{\alpha}: \quad(-1 / \mathrm{m})\left[D_{a}+\left(\partial D_{a} / \partial \alpha\right)\right]$

$Z_{q}: \quad(-1 / \mathrm{m})(\partial L / \partial Q)$

$Z_{V}: \quad(-1 / \mathrm{m})\left(\partial L / \partial V_{T}\right)$.

\section{Conflict of Interests}

The authors declare that there is no conflict of interests regarding the publication of this paper.

\section{Acknowledgments}

Fany Mendez-Vergara and Angelica Mendoza-Torres acknowledge financial support from CONACYT. Ilse Cervantes and Angelica Mendoza-Torres acknowledge financial support from CONACYT-FORDECYT Grant no. 190966. The authors acknowledge technical support from Irwin Allen Diaz-Diaz.

\section{References}

[1] D. J. Leith and W. E. Leithead, "Survey of gain-scheduling analysis and design," International Journal of Control, vol. 73, no. 11, pp. 1001-1025, 2000.

[2] D. J. Stilwell, "State-space interpolation for a gain-scheduled autopilot," Journal of Guidance, Control, and Dynamics, vol. 24, no. 3, pp. 460-465, 2001.

[3] B. P. Rasmussen and Y. J. Chang, "Stable controller interpolation and controller switching for LPV systems," Journal of Dynamic Systems, Measurement and Control, vol. 132, no. 1, Article ID 011007, 12 pages, 2010.

[4] G. Cai, C. Hu, B. Yin, H. He, and X. Han, "Gain-scheduled $\mathrm{H}_{2}$ controller synthesis for continuous-time polytopic LPV systems," Mathematical Problems in Engineering, vol. 2014, Article ID 972624, 14 pages, 2014.

[5] D. J. Leith and W. E. Leithead, "Gain-scheduled control: relaxing slow variation requirements by velocity-based design," Journal of Guidance, Control, and Dynamics, vol. 23, no. 6, pp. 988-1000, 2000.

[6] D. Enns, D. Bugajskia, R. Hendricka, and G. Stein, "Dynamic inversion: an envolving methodology for flight control design," International Journal of Control, vol. 59, no. 1, pp. 71-91, 1994.

[7] A. Packard, "Gain scheduling via linear fractional transformations," Systems \& Control Letters, vol. 22, no. 2, pp. 79-92, 1994.

[8] P. Apkarian, P. Gahinet, and G. Becker, "Self-scheduled $H_{\infty}$ control of linear parameter-varying systems: a design example," Automatica, vol. 31, no. 9, pp. 1251-1261, 1995.

[9] W. J. Rugh and J. S. Shamma, "Research on gain scheduling," Automatica, vol. 36, no. 10, pp. 1401-1425, 2000.

[10] A. Ataei and Q. Wang, "Non-linear control of an uncertain hypersonic aircraft model using robust sum-of-squares method," IET Control Theory \& Applications, vol. 6, no. 2, pp. 203-215, 2012.

[11] M. T. Frye, S. Ding, C. Qian, and S. Li, "Fast convergent observer design for output feedback stabilisation of a planar vertical takeoff and landing aircraft," IET Control Theory \& Applications, vol. 4, no. 4, pp. 690-700, 2010.

[12] B. Zhu, X. Wang, and K.-Y. Cai, “Tracking control for angularrate-sensorless vertical take-off and landing aircraft in the presence of angular-position measurement delay," IET Control Theory \& Applications, vol. 4, no. 6, pp. 957-969, 2010.

[13] R. Zhang, Q. Quan, and K.-Y. Cai, "Attitude control of a quadrotor aircraft subject to a class of time-varying disturbances," IET Control Theory \& Applications, vol. 5, no. 9, pp. 1140-1146, 2011.

[14] R. Kamyar and E. Taheri, "Aircraft optimal terrain/threat-based trajectory planning and control," Journal of Guidance, Control, and Dynamics, vol. 37, no. 2, pp. 466-483, 2014.

[15] Q. Hu, B. Xiao, D. Wang, and E. K. Poh, "Attitude control of spacecraft with actuator uncertainty," Journal of Guidance, Control, and Dynamics, vol. 36, no. 6, pp. 1771-1776, 2013.

[16] A. H. J. de Ruiter, "Spacecraft attitude tracking with guaranteed performance bounds," Journal of Guidance, Control, and Dynamics, vol. 36, no. 4, pp. 1214-1221, 2013.

[17] J. Hu and H. Zhang, "Bounded output feedback of rigid-body attitude via angular velocity observers," Journal of Guidance, Control, and Dynamics, vol. 36, no. 4, pp. 1240-1248, 2013.

[18] Q. Sang and G. Tao, "Multivariable adaptive piecewise linear control design for NASA generic transport model," Journal of Guidance, Control, and Dynamics, vol. 5, no. 35, pp. 1559-1567, 2012.

[19] P. Casau, D. Cabecinhas, and C. Silvestre, "Hybrid control strategy for the autonomous transition flight of a fixed-wing aircraft," IEEE Transactions on Control Systems Technology, vol. 6, no. 21, pp. 2194-2211, 2013.

[20] Y. Hou, Q. Wang, and C. Dong, "Gain scheduled control: switched polytopic system approach," Journal of Guidance, Control, and Dynamics, vol. 34, no. 2, pp. 623-628, 2011.

[21] I. Hwang, H. Balakrishnan, and C. Tomlin, "State estimation for hybrid systems: applications to aircraft tracking," IEE Proceedings. Control Theory \& Applications, vol. 153, no. 5, pp. 556-566, 2006.

[22] L. G. Sun, C. C. de Visser, Q. P. Chu, and Q. P. Falkena, "Hybrid sensor-based backstepping control approach with its application to fault-tolerant flight control," Journal of Guidance, Control, and Dynamics, vol. 1, no. 37, pp. 59-71, 2014. 
[23] J. Roskam, Airplane Flight Dynamics and Automatic Flight Controls. Part I, John Wiley \& Sons, 3rd edition, 1995.

[24] A. N. Kolmogorov and S. V. Formin, Elements of the Theory of the Functional Analysis-I: Metric and Normed Sapaces, Courier Dover Publications, Mineola, NY, USA, 1957.

[25] H. Kimura, "Pole assignment by gain output feedback," IEEE Transactions on Automatic Control, vol. 20, no. 4, pp. 509-516, 1975.

[26] P. Kokotović, H. K. Khalil, and J. O'Reilly, Singular Perturbation Methods in Control: Analysis and Design, vol. 25, Prentice Hall, Englewood Cliffs, NJ, USA, 3rd edition, 1999.

[27] F. C. Hoppensteadt, "Singular perturbations on the infinite interval," Transactions of the American Mathematical Society, vol. 123, no. 2, pp. 521-535, 1966.

[28] J. P. Hespanha, D. Liberzon, D. Angeli, and E. D. Sontag, "Nonlinear norm-observability notions and stability of switched systems," IEEE Transactions on Automatic Control, vol. 50, no. 2, pp. 154-168, 2005.

[29] B. L. Stevens and F. L. Lewis, Aircraft Control and Simualtion, John Wiley \& Sons, New York, NY, USA, 2nd edition, 2003. 


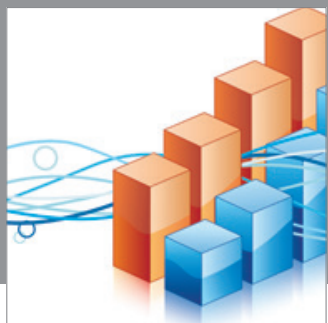

Advances in

Operations Research

mansans

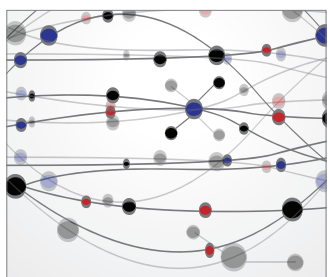

The Scientific World Journal

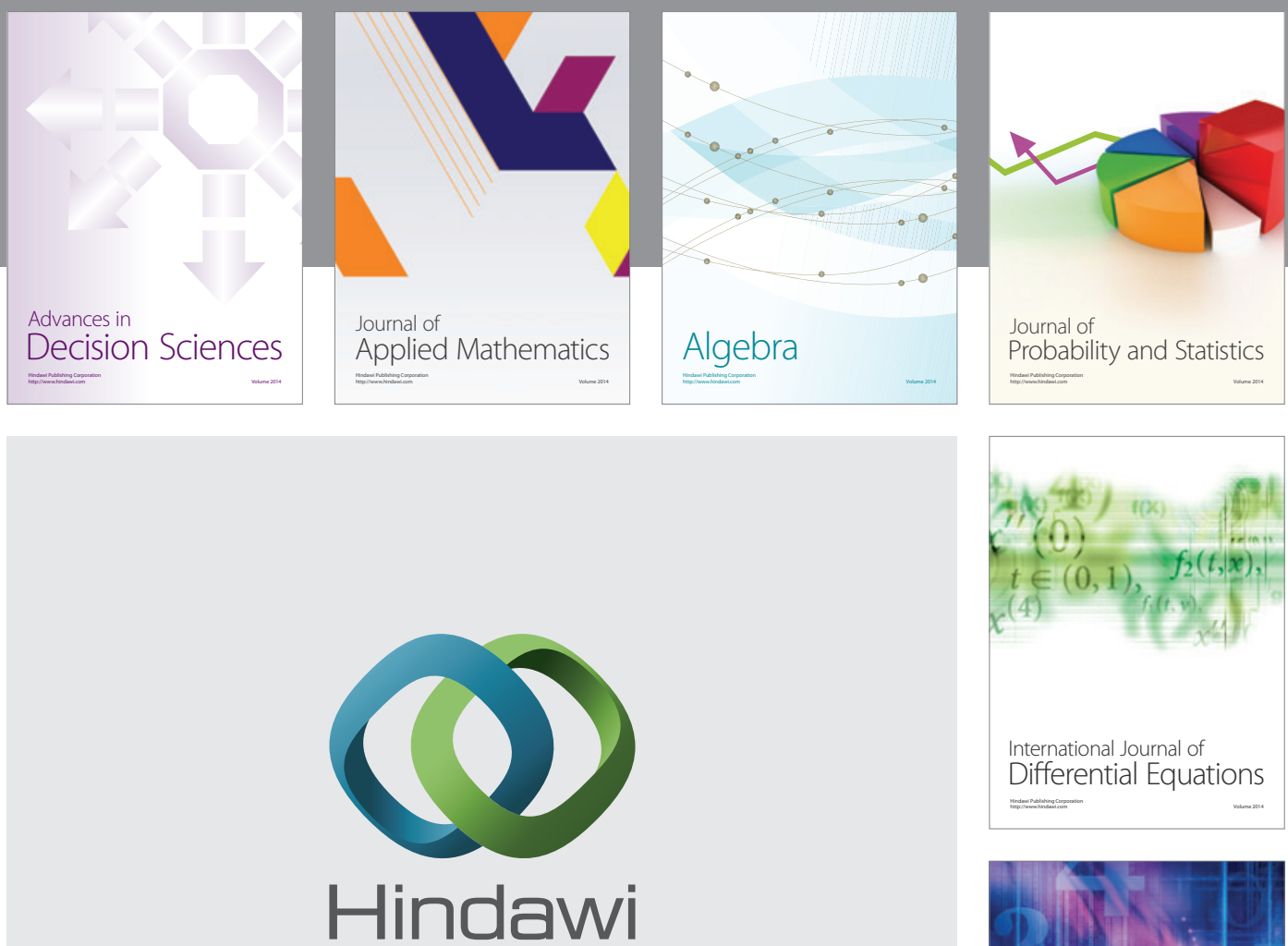

Submit your manuscripts at http://www.hindawi.com
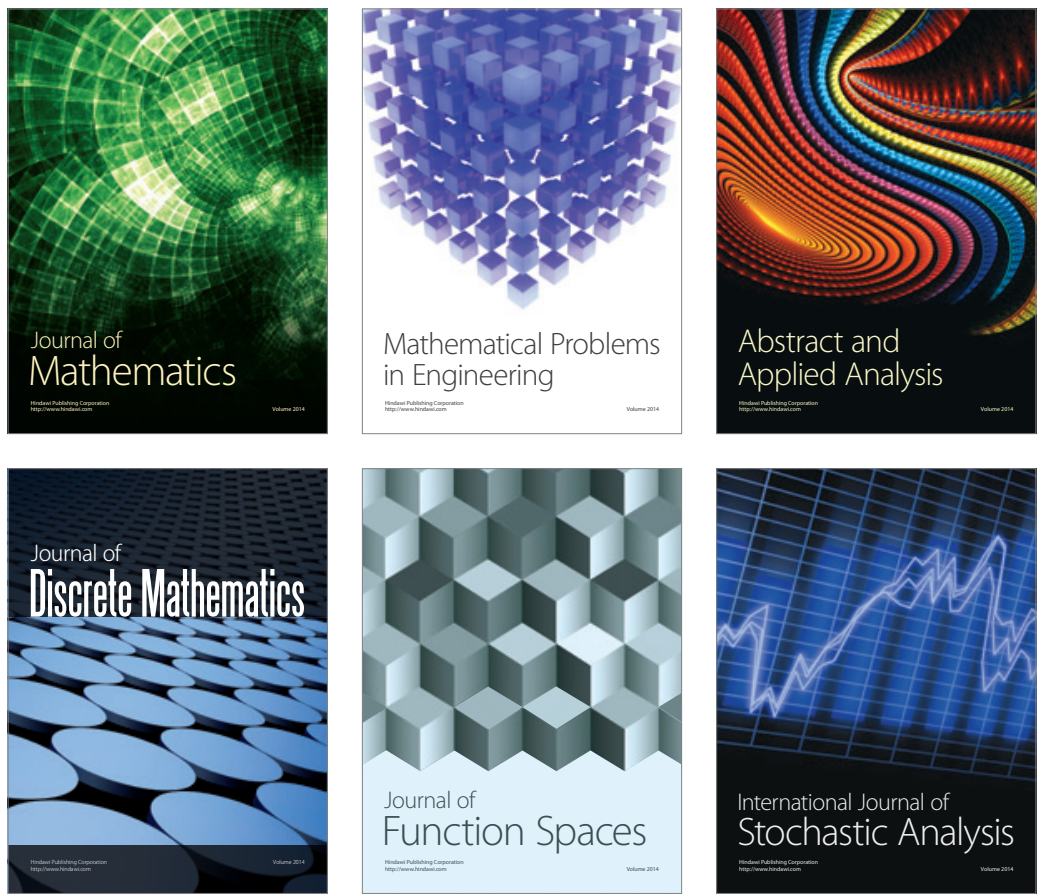

Journal of

Function Spaces



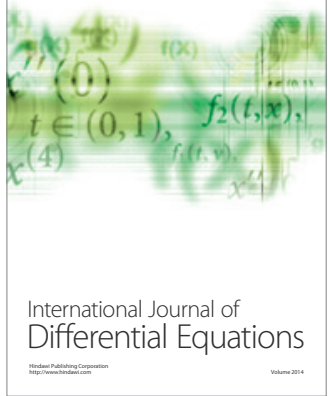
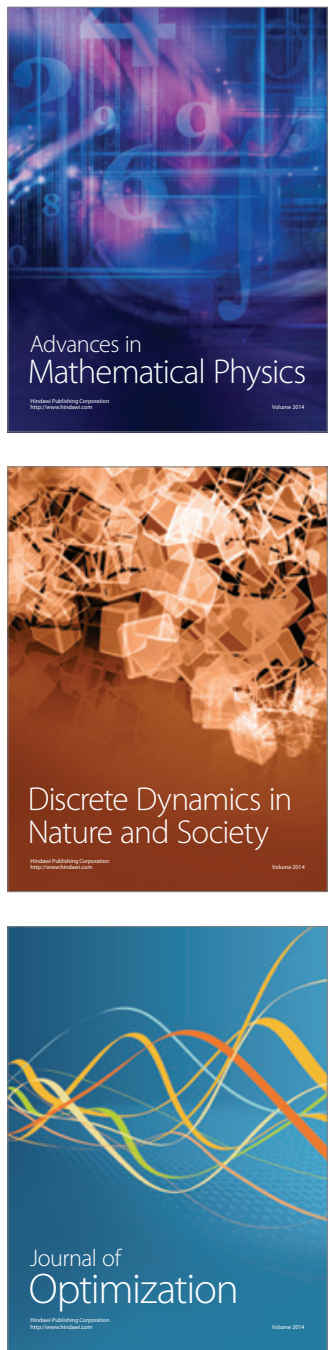